\title{
A Patient in Whom Straightening of a Report Parent Vessel Related to Insertion of a Braided Stent Contributed to Complete Occlusion of an Intracranial Aneurysm
}

Takashi Fujii, ${ }^{1}$ Hidenori Oishi, ${ }^{1}$ Kohsuke Teranishi, ${ }^{2}$ Kenji Yatomi, ${ }^{2}$ and Munetaka Yamamoto ${ }^{2}$

Objective: We report a patient in whom vascular straightening was achieved after stent-assisted coil embolization, leading to complete occlusion of an intracranial aneurysm after 1 year.

Case Presentation: The patient was a 60-year-old female. A medical checkup of the brain showed a posterior inferior cerebellar artery (PICA) aneurysm. Under general anesthesia, coil embolization was performed. During surgery, a coil deviated onto the PICA side, and a stent was deployed so that the aneurysmal neck might be located at its center. Finally, incomplete occlusion of the aneurysm was achieved. Cerebral angiography 1 year after surgery indicated a sharper branching angle of the blood vessel in comparison with the preoperative angle and complete occlusion of the aneurysm. Conclusion: A braided stent inserted to a site where a thin parent vessel is not fixed by the peripheral structure may make the parent vessel straight, contributing to complete occlusion of an aneurysm.

Keywords > stent assist coiling, aneurysm, straightening parent vessel, low-profile visualized intraluminal support junior

\section{Introduction}

Several studies reported that vessel straightening after stent-assisted coil embolization led to complete occlusion of aneurysms despite incomplete occlusion immediately after surgery. In most studies, an Enterprise stent (Codman Neurovascular, Raynham, MA, USA) was used. To our knowledge, no study has reported complete occlusion of cerebral aneurysms resulting from vascular straightening related to the insertion of a low-profile visualized intraluminal support (LVIS) stent (Terumo Corporation, Tokyo, Japan). In this study, we report a patient in whom

\footnotetext{
${ }^{1}$ Department of Neuroendovascular Therapy, Juntendo University Faculty of Medicine, Tokyo, Japan

${ }^{2}$ Department of Neurosurgery, Juntendo University Faculty of Medicine, Tokyo, Japan
}

Received: February 27, 2018; Accepted: July 17, 2018

Corresponding author: Takashi Fujii. Department of Neuroendovasular Therapy, Juntendo University Faculty of Medicine, 2-1-1 Hongo, Bunkyo-ku, Tokyo 113-8421, Japan

Email: takashigreengreen@yahoo.co.jp

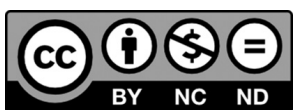

This work is licensed under a Creative Commons Attribution-NonCommercialNoDerivatives International License.

C2019 The Japanese Society for Neuroendovascular Therapy stent-assisted coil embolization of an unruptured posterior inferior cerebellar artery (PICA) aneurysm was performed using a low-profile visualized intraluminal support junior (LVIS Jr) stent (Terumo), and cerebral angiography after 1 year showed complete occlusion, and review the literature.

\section{Case Presentation}

The patient was a 60 -year-old female. A medical checkup of the brain showed an unruptured cerebral aneurysm. She was referred to our hospital for detailed examination and treatment. On the initial consultation, there were no abnormal neurologic findings. She had a history of hypertension. Her family history was not contributory. Angiography revealed a relatively wide-neck aneurysm measuring $4.5 \mathrm{~mm}$ in maximum diameter and $3.8 \mathrm{~mm}$ in neck diameter in the anterior medullary segment of the PICA (Fig. 1A-1C). Under general anesthesia, coil embolization was performed. An Axcelguide 6 Fr 80 cm STR (Medikit co. ltd., Tokyo, Japan) was inserted into the proximal left vertebral artery (VA) through the right femoral artery. A Cerulean G 6 Fr $105 \mathrm{~cm}$ STA (Medikit) was inserted into the proximal VA. An Excelsior SL-10 2M (Stryker, Fremont, CA, USA) was guided into the aneurysm using a GT wire 0.012 inch double angle (Terumo). Coil embolization was performed, but 

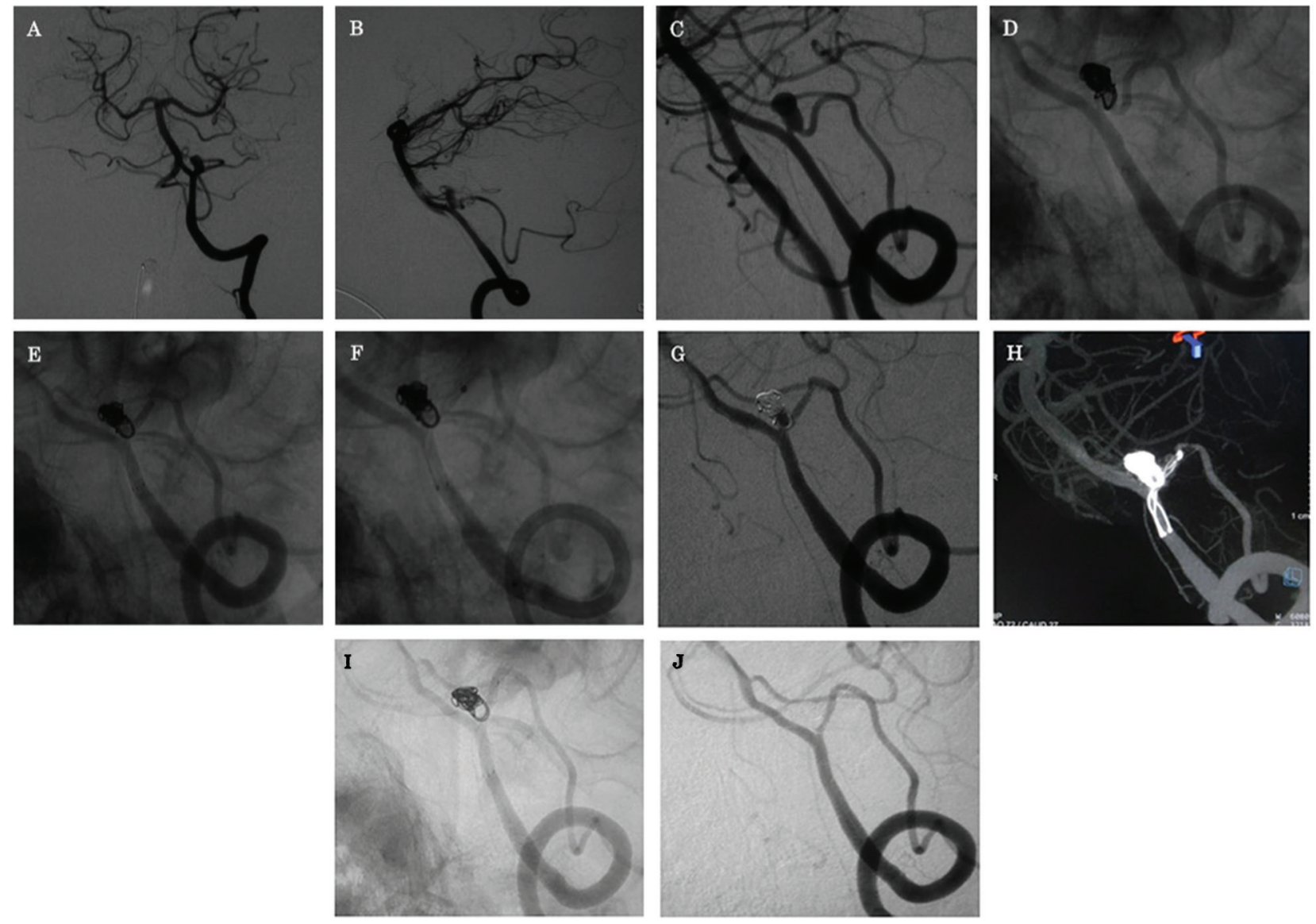

Fig. 1 (A-C) Preoperative DSA of the left VA showed a relatively wide-neck aneurysm measuring $4.5 \mathrm{~mm}$ in maximum diameter and $3.8 \mathrm{~mm}$ in neck diameter in the anterior medullary segment of the PICA. There was no bleb, but its shape was slightly irregular. (A) AP view, (B) lateral view, (C) best angle. (D) DA of the left VA during coil embolization showed a deviation from the coil mass into the one-loop parent vessel, PICA. (E) DA of the left VA immediately after deploying an LVIS Jr stent (Terumo Corporation, Tokyo, Japan) indicated insufficient stent dilation. (F) Left VA angiography after PTA with a Hyperform (eV3 Neurovascular, Irvine, CA, USA) confirmed favorable stent dilation. (G) DSA of the left VA at the completion of surgery showed incomplete occlusion of the aneurysm. (H) Cone-beam CT confirmed adequate stent deployment. (I) DA of the left VA 1 year after surgery confirmed a sharp bifurcation angle between the PICA and VA related to stenting. (J) DSA of the left VA 1 year after surgery confirmed complete occlusion of the aneurysm. AP: anteriorposterior; DA: digital angiography; LVIS Jr: low-profile visualized intraluminal support junior; PICA: posterior inferior cerebellar artery; PTA: percutaneous transluminal angioplasty; VA: vertebral artery

a coil deviated onto the PICA side (Fig. 1D), and a Headway 17STR 2M (Terumo) was guided to the distal area of the PICA using a CHIKAI 0.014 inch $200 \mathrm{~cm}$ (Asahi Intecc, Aichi, Japan) to preserve the PICA. An LVIS Jr stent measuring $2.5 \times 13 \mathrm{~mm}$ was deployed from the PICA to the VA so that the aneurysmal neck might be located at its center (Fig. 1E). As stent dilation was insufficient, the stent was dilated using a Hyperform $4 \times 7 \mathrm{~mm}(\mathrm{eV} 3 \mathrm{Neu}-$ rovascular, Irvine, CA, USA) (Fig. 1F). Finally, the procedure was completed, with body filling (Fig. 1G). At the completion of surgery, cone-beam CT was performed to confirm adequate stent deployment (Fig. 1H). After 1 year, angiography showed complete occlusion of the cerebral aneurysm (Fig. $\mathbf{1}$ and $\mathbf{1 J}$ ).

\section{Discussion}

For stent-assisted coil embolization of cerebral aneurysms, three stents: Neuroform (Stryker), Enterprise, and LVIS stents, have been approved as devices that facilitate tight packing while preventing coil deviation into a parent blood vessel. Several studies reported their usefulness. ${ }^{1-4)}$ The metallic coverage rate of an LVIS stent is $28 \%$, being higher than those of the other two stents. According to some case reports, potent embolization effects were obtained through flow diversion (FD) effects (metallic coverage rate: LVIS Jr, 18\%; Neuroform, 11\%; and Enterprise, 10\%). A consensus regarding the most appropriate stent has not been reached, and these stents are selected 
based on a characteristics of respective stent or a preference of surgeon. ${ }^{2,4,5)}$

PICA aneurysms are adjacent to the inferior cranial nerves or medulla oblongata at their periphery, and surgical clipping is difficult. On the other hand, for endovascular treatment, PICA-involved-type aneurysms at the VA-PICA bifurcation or wide-neck aneurysms require adjunctive techniques with a balloon or stent. ${ }^{6}$ ) The PICA is thin as a blood vessel into which a stent is inserted, and the usefulness of treatment with an LVIS Jr stent was reported.7)

A study examined the results of coil embolization with respect to the presence or absence of stent use in patients in whom incomplete occlusion of cerebral aneurysms at the completion of coil embolization led to complete occlusion. ${ }^{8)}$ Although there was no significant difference between stentassisted and non-stent-assisted groups, the use of stents markedly increased the complete occlusion rate, excluding confounding factors, such as the aneurysmal size.

Previous studies indicated that the insertion of an Enterprise stent facilitated vascular straightening, contributing to aneurysmal occlusion through hemodynamic changes. ${ }^{6,9,10)}$ Takemoto et al. $\left.{ }^{9}\right)$ reported a patient in whom the insertion of an Enterprise stent led to vessel straightening, resulting in complete occlusion of an aneurysm. Concerning the mechanism, these studies suggested that stent-insertionrelated vascular endothelium formation involving microaneurysms contributes to complete occlusion in addition to vessel straightening. In the present case, percutaneous transluminal angioplasty (PTA) with a balloon was performed after stent insertion. For vascular intimal neogenesis, the vascular endothelial disorder related to PTA or stent's radial force $(\mathrm{RF})$ is required. In the present case, balloon dilation after stent insertion may have induced subsequent vascular intimal neogenesis, contributing to complete occlusion. ${ }^{11}$ ) According to case reports of treatment with a stent alone, as described above, treatment with a Neuroform stent led to incomplete occlusion, whereas the results of balloondilation-type stent insertion were favorable. ${ }^{12,13)}$

Interestingly, Hirdes et al. ${ }^{14)}$ compared stent performance. They investigated the radial and axial forces (RF and AF, respectively) of autodilating esophageal stents. They indicated that there was a negative correlation between the RF and AF of esophageal stents. Briefly, the AF of stents with a high RF was low, whereas the RF of stents with a high AF was low. In addition, when comparing a braided stent (BS) with a laser cut stent (LCS), the AF of the BS was high, and the RF of the LCS was high. Cho et al. ${ }^{15)}$ compared the performance of four intracranial stents: LVIS, LEO (Balt,
Montmorency, France), Enterprise, and Neuroform stents. They reported that the RF of an LVIS stent was the weakest among the four stents, whereas its AF was the strongest. Various studies indicated stent-insertion-related changes in computational fluid dynamic (CFD) analysis. ${ }^{12,16,17)}$ The FD effects of an LVIS stent are marked, and they are more marked than those of overlapping Enterprise stents. In addition, the FD effects of overlapping LVIS stents are more potent than those of a Pipeline stent. ${ }^{16)}$ Gao et al. reported that a sharp vascular bifurcation angle related to stenting significantly reduced the wall shear stress (WSS) for the aneurysmal neck. ${ }^{17)}$ In addition, another study indicated that changes in the vascular bifurcation angle after LVIS insertion were more marked than after Enterprise insertion. ${ }^{2)}$

In the present case, a change in the vascular bifurcation angle after the insertion of an LVIS Jr stent may have contributed to complete occlusion of the aneurysm. On the other hand, the FD effects of an LVIS stent are more marked than those of conventional stents, and occlusion associated with PTA-related vascular intimal neogenesis may be achieved. Thus, various factors are involved in occlusion of aneurysms. To clarify the involvement of vascular bifurcation angle changes in occlusion of aneurysms, a larger number of patients should be investigated in the future.

Internationally, a Flow Re-Direction Endoluminal Device Junior (FRED Jr, MicroVension, Tustin, CA, USA) is used for the treatment of cerebral microaneurysms although it is not covered by health insurance in Japan. Favorable results have been obtained. ${ }^{18)}$ Such new devices may be applied in the future.

\section{Conclusion}

We encountered a patient in whom stent-assisted coil embolization of a PICA aneurysm was performed, and incomplete occlusion was achieved immediately after surgery, but angiography after 1 year showed complete occlusion. Stent-assisted coil embolization of an aneurysm derived from a thin parent vessel, as demonstrated in the present case, using an LVIS Jr stent may contribute to complete occlusion.

\section{Disclosure Statement}

Hidenori Oishi as the representative of a donated fund laboratory, received a donation of $\geq 20000000$ yen/year from Terumo Corporation. There is no conflict of interest for the other coauthors. 


\section{References}

1) Cho YD, Sohn CH, Kang HS, et al: Coil embolization of intracranial saccular aneurysms using the low-profile visualized intraluminal support (LVISTM) device. Neuroradiology 2014; 56: 543-551.

2) Ge H, Lv X, Yang X, et al: LVIS stent versus enterprise stent for the treatment of unruptured intracranial aneurysms. World Neurosurg 2016; 91: 365-370.

3) Johnson AK, Munich SA, Tan LA, et al: Complication analysis in nitinol stent-assisted embolization of 486 intracranial aneurysms. $J$ Neurosurg 2015; 123: 453-459.

4) Durst CR, Khan P, Gaughen J, et al: Direct comparison of Neuroform and Enterprise stents in the treatment of widenecked intracranial aneurysms. Clin Radiol 2014; 69: e471-e476.

5) Wang J, Vargas J, Spiotta A, et al: Stent-assisted coiling of cerebral aneurysms: a single-center clinical and angiographic analysis. J Neurointerv Surg 2018; 10: 687-692.

6) Cho YD, Kang HS, Lee WJ, et al: Stent-assisted coil embolization of wide-necked posterior inferior cerebellar artery aneurysms. Neuroradiology 2013; 55: 877-882.

7) Samaniego EA, Abdo G, Hanel RA, et al: Endovascular treatment of PICA aneurysms with a low-profile visualized intraluminal support (LVIS Jr) device. J Neurointerv Surg 2016; 8: 1030-1033.

8) Jeon JP, Cho YD, Rhim JK, et al: Effect of stenting on progressive occlusion of small unruptured saccular intracranial aneurysms with residual sac immediately after coil embolization: a propensity score analysis. $J$ Neurointerv Surg 2016; 8: 1025-1029.

9) Takemoto K, Tateshima S, Rastogi S, et al: Disappearance of a small intracranial aneurysm as a result of vessel straightening and in-stent stenosis following use of an Enterprise vascular reconstruction device. J Neurointerv Surg 2014; 6: e4.
10) Gao B, Baharoglu MI, Cohen AD, et al: Y-stent coiling of basilar bifurcation aneurysms induces a dynamic angular vascular remodeling with alteration of the apical wall shear stress pattern. Neurosurgery 2013; 72: 617-629; discussion 628-629.

11) Wakhloo AK, Mandell J, Gounis MJ, et al: Stent-assisted reconstructive endovascular repair of cranial fusiform atherosclerotic and dissecting aneurysms: long-term clinical and angiographic follow-up. Stroke 2008; 39: 3288-3296.

12) Zenteno MA, Santos-Franco JA, Freitas-Modenesi JM, et al: Use of the sole stenting technique for the management of aneurysms in the posterior circulation in a prospective series of 20 patients. J Neurosurg 2008; 108: 1104-1118.

13) Zenteno MA, Murillo-Bonilla LM, Guinto G, et al: Sole stenting bypass for the treatment of vertebral artery aneurysms: technical case report. Neurosurgery 2005; 57: E208; discussion E208.

14) Hirdes MM, Vleggaar FP, de Beule M, et al: In vitro evaluation of the radial and axial force of self-expanding esophageal stents. Endoscopy 2013; 45: 997-1005.

15) Cho SH, Jo WI, Jo YE, et al: Bench-top comparison of physical properties of 4 commercially-available self-expanding intracranial stents. Neurointervention 2017; 12: 31-39.

16) Wang C, Tian Z, Liu J, et al: Flow diverter effect of LVIS stent on cerebral aneurysm hemodynamics: a comparison with Enterprise stents and the Pipeline device. J Transl Med 2016; 14: 199.

17) Gao B, Baharoglu MI, Malek AM: Angular remodeling in single stent-assisted coiling displaces and attenuates the flow impingement zone at the neck of intracranial bifurcation aneurysms. Neurosurgery 2013; 72: 739-748; discussion 748 .

18) Möhlenbruch MA, Kizilkilic O, Killer-Oberpfalzer M, et al: Multicenter experience with FRED Jr flow re-direction endoluminal device for intracranial aneurysms in small arteries. AJNR Am J Neuroradiol 2017; 38: 1959-1965. 